ISSUES IN MEDICINE

\title{
Red meat, processed meat and cancer in South Africa
}

\author{
D C Stefan
}

Cristina Stefan, $M D, M M e d, F C P, C M O, M S c, P h D$, is a paediatric oncologist and cancer epidemiologist. As the first woman president-elect of the African Organization for Research and Training in Cancer, she is deeply involved in African and global cancer activities. She is vice-president of the South African Medical Research Council.

Corresponding author: D C Stefan (cristina.stefan@mrc.ac.za)

Epidemiological studies around the world were analysed recently by the International Agency for Research on Cancer, demonstrating a positive correlation between consumption of red meat and processed meat and colorectal cancer. In South Africa (SA) there is a great variation in the incidence of this type of cancer between various ethnic groups, related to diet and other risk factors. Strengthening the SA cancer registry and co-ordinated research on diet and cancer are required to provide specific answers for our population.

S Afr Med J 2016;106(1):43. DOI:10.7196/SAMJ.2016.v106i1.10400

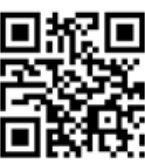

The most recent South African (SA) cancer registry analysis, from $2010,{ }^{[1]}$ shows that colorectal cancer is in seventh position for males, with a total number of 1291 new cases, after basal cell carcinoma, prostate cancer, squamous cell carcinoma of the skin, primary site unknown, Kaposi's sarcoma and lung carcinoma. The same cancer is in sixth position for females, with a total number of 1129 new cases, after breast cancer, cervical cancer, basal cell carcinoma, squamous cell carcinoma of the skin and primary site unknown. The lifetime ( 0 - 74 years) risk of developing colorectal cancer is $1 / 114$ for males and $1 / 182$ for females in SA.

There is a considerable variation in the incidence of diagnosed colorectal cancers in the different ethnic groups, possibly as a result of dietary differences, among other factors that need to be considered.

Among SA Asian males colorectal cancer was the second most common histologically confirmed cancer in 2010, accounting for $13.62 \%$ of total cancers (just after prostate cancer). It was also the second most common cancer among Asian females, accounting for $7.39 \%$ of total cancers, just after breast cancer. However, histologically confirmed colorectal cancer accounted for only 3.84\% of total cancers (after adjustments) in the black male population, and for only $2.54 \%$ in black females, much lower than in the other groups. White females had a higher percentage of new histologically diagnosed colorectal cancers in 2010 than white males $(5.17 \% \mathrm{v}$. $4.83 \%$ of all malignancies).

The incidence of pancreatic cancer in the SA population is low or not reported, and that of cancer of the prostate differs significantly among the various population groups.

A considerable amount of research indicates that consumption of red meat is associated with an increased risk of cancer. The International Agency for Research on Cancer (IARC) will shortly publish a monograph on the epidemiological studies addressing the correlation between red meat consumption and cancer in humans. ${ }^{[2]}$ Lancet Oncology of 26 October 2015 presented the IARC-analysed data on the association of red meat consumption with colorectal cancer. ${ }^{[3]} \mathrm{A}$ large section of the data originates in 14 cohort studies, done over 20 years. Positive associations were seen with high v. low consumption of red meat in half of those studies, including a cohort from ten European countries covering a wide range of meat consumption, and other large cohorts from Sweden and Australia.
According to the IARC, studies around the world found 'sufficient evidence in humans that the consumption of processed meat causes colorectal cancer. The international organisation defines processed meat as any type of meat that is salted, cured, fermented or smoked to enhance its flavour or preserve it. Processed meat generally contains pork or beef, but may also contain poultry. In addition, the working group from the same agency concluded that there is limited evidence in human beings for the carcinogenicity of the consumption of unprocessed red meat, which was mainly associated with colon cancer but also with pancreatic and prostate cancer.

Since no data from Africa were included, it is important to assess the situation in SA.

While the IARC data on the carcinogenic risk of red meat are strong, their application to the SA context requires careful consideration. SA is a country with a diverse population in which genetic factors, diet, cultural traditions, lifestyle and exercise contribute to various extents to the differences reported. The wide difference between the highest and the lowest income brackets may also have a substantial influence on the incidence of colorectal, prostate, pancreatic and the other types of cancers in question, because of its impact on food consumption patterns,

In the quest for understanding the risk factors for cancer in our country, the need for an updated and comprehensive cancer registry cannot be sufficiently emphasised. Nevertheless, co-ordinated research into the epidemiology, risk factors (including dietary constituents) and prevention of cancer should be the key factor in establishing the national cancer control strategy.

Until more evidence based on research into local conditions is available, the IARC findings, which support 'recommendations to limit intake of meat', particularly in processed forms, should be considered in the context of promoting a healthy, balanced diet consisting mainly of vegetables and fruit, with limited sugar and alcohol consumption, as well as encouraging physical activity.

\footnotetext{
National Institute for Occupational Health. Cancer in South Africa, 2010. National Cancer Registry. http://www.nioh.ac.za/assets/files/NCR_Final_2010_tables(1).pdf (accessed 26 November 2015).

2. International Agency for Research on Cancer. Press release No. 240. IARC monographs evaluate consumption of red meat and processed meat. https://www.iarc.fr/en/media-centre/pr/2015/pdfs/ pr240_E.pdf (accessed 26 November 2015).

3. Bouvard V, Loomis D, Guyton KZ, et al. Carcinogenicity of consumption or red and processed meat. Lancet Oncol 2015;16(15):1599-1600. [http://dx.doi.org/10.1016/S1470-2045(15)00444-1]
} 